\title{
Adaptive EIS with Business Rules Discovered by Formal Concept Analysis
}

\author{
Victor Romanov $^{1}$, Alina Poluektova ${ }^{1}$, and Olga Sergienko ${ }^{2}$ \\ ${ }^{1}$ Russian Plekhanov University, Russia, Moscow \\ \{victorromanov1, poluektovaau\} @gmail.com \\ ${ }^{2}$ Software Group IBM East Europe/Asia \\ Russia, Moscow \\ Sergienko@ru.ibm.com
}

\begin{abstract}
Business rules component became essential part of such software as ORACLE, SAP, IBM and Microsoft and this fact signifies new stage in the Enterprise Information Systems development and applications. The efficiency of application such new tools depends from business rules development technology. The new generation of EIS software requires not only deployment strategy, but also tools for extracting business rules from description of existing practice. Along with manual business rules extraction from mountains of documents there exists the possibility to apply data mining technology based on formal concept analysis. In this paper we are presenting, how supplier and customers data, being accumulated in data base, may be used in CRM system for fitting services and relations to customer and supplier profile.
\end{abstract}

Keywords: Enterprise Information systems, business rules, formal concept analysis, system adaptation, customer profile.

\section{$1 \quad$ Introduction}

The modern enterprises' business processes are very complex, especially for medium and large business. They contain many conditions, restrictions and rules that are implicit and hidden in numerous documents, job manuals, applications codes and experience of employees. Such sparse rules dissemination creates difficulties to company to rebuild the business in time, because of time spending for documents finding, the conditions and rules reveal, rewriting instructions and regulations, and to make changes to the IT components and applications. Great number of business rules and its variability urges the companies to allocate business rules as independent part of the business description [1]. The collecting business rules together as a separate IT components managed by Business Rules Management System increases the response speed to the changing of competitive environment and speed of decision making.

The companies, such as Oracle, SAP, IBM and Microsoft, which are producing software for enterprise information systems during last two years the main effort have been focused at the problems of integrating broad spectrum of the different their 
software products, such as Web-applications, SOA, Collaboration suite, Data Warehousing and so on. In the same time such business intelligence tools as Data Mining, Knowledge Discovery, and Business rules were regarded for a long time how not essential part or as special software and even were not included into structure of EIS software.

So there arose a situation, when very complex and expensive software was oriented on the secondary tasks in the business management, that is on the routine tasks of data processing, not on the tasks of top managers for strategic planning. As a result in many cases the efficiency of enterprise information systems implementation being compared with expenses was not sufficient and did not satisfies users.

Meanwhile the concepts of EDM - Enterprise Decision Management [2] and ADS -Automated Decision Support [3] were proposed as an approach for possible automated decision making. Having in mind implementation business rules approach at the some enterprise, we will concentrate our efforts on the problem of including business rule capability on CRM business processes. Only in recent years the companies mentioned have included a component of business rules in their software. It is important to note that rule-component has been integrated into business processes component and Data miming tools are applying for rule discovery. Let's consider some essential features of BRMS components EIS software of these vendors.

\section{BRMS Component of the Leading EIS SOFTWARE Companies}

\subsection{Oracle Business Rules}

Oracle Business Rules [4] is a new product that provides all features needed to realize the "agility" and cost reduction benefits of Business Rules. Oracle Data Mining can analyze historical transaction data and suggest business rules. Oracle integrate Business Rules integrates with SOA/BPM facilities.

The Rule Author is a Graphical User Interface tool for creating and updating Rules. The programmers use the Rules Author to create a business rules, extracting it from documents and practice and converting these business terms to Java or XML expressions. Rule Author provides a web-based graphical environment that enables the easy creation of business rules via a web browser.

The Rules engine is implemented as a Java Class, and is deployed as a Java callable library. Java programs directly call the Rules engine. The Rules engine implements the industry standard Rete algorithm making it optimized for efficiently processing large numbers of Rules. There are many types of services including "decision support" services.

\subsection{SAP NetWeaver Business Rules Management}

The SAP NetWeaver Business Rules Management [5] component complements and accelerates SAP NetWeaver Business Process Management. Together they have become important components of Enterprise SOA. 
The Rules Composer is the rule modeling and implementation environment of SAP NetWeaver BRM. Because it is integrated within SAP NetWeaver Developer Studio, the Rules Composer is the most efficient way for developers to build rules-based applications targeted at the SAP NetWeaver platform.

The Rules Engine is the run-time engine of SAP NetWeaver BRM, available as a predeployed stateless session bean in the SAP NetWeaver Application Server (SAP NetWeaver AS) Java of SAP NetWeaver CE. This tool gives IT developers the ability to generate reusable rules services out of the box, which is particularly helpful for integrating rules into composite applications.

The Rules Analyzer, a targeted environment for business analysts, will allow them to model, test, simulate, and analyze business rules without assistance from developers

\subsection{MS BizTalk Server}

BizTalk Server includes the Business Rules Framework [6] as a stand-alone .NETcompliant class library that includes a number of modules, support components, and tools. The primary modules include the Business Rule Composer for constructing policies, the Rule Engine Deployment Wizard for deploying policies created in the Business Rule Composer, and the Run-Time Business Rule Engine that executes policies on behalf of a host application.

The Business Rule Composer enables you to create rules by adding predicates and facts and defining actions. You can add facts and actions by dragging them to the Business Rule Composer design surface. The actions update the nodes in the specified document. You can also add AND, OR, and NOT operators to conditions to create complex comparisons.

The Business Rule Composer helps you create, test, publish, and deploy multiple versions of business rule policies and vocabularies to make the management of these artifacts easier.

\section{$2.4 \quad$ IBM ILog JRules}

Rule Studio JRules and Rules for .NET [7] provide a rule application development environment called Rule Studio. From within Rule Studio, a developer can:

- Create a logical business object model (BOM) for the application, and map it to a customized, domain-specific rule vocabulary.

- Create business rules in a natural language syntax, which can be expressed in one or a more localized versions (for example, English or Spanish).

- Create rules in the form of decision tables and decision trees

- Create technical rules in a platform-specific syntax.

- Separate rules in a rule set into tasks, and specify a rule flow to orchestrate the execution of these tasks. 
The rule authoring and management environment for business analysts and policy managers is called IBM WebSphere ILOG Rule Team Server (RTS), a thin-client Web-based environment with a scalable, high-performance enterprise rule repository. The repository provides the BRMS with a central "source of truth", addressing the specific needs of rule-based business policy management.

The WebSphere ILOG BRMS offerings include Rule Execution Server (RES), a managed, monitorable execution environment for rules that can be incorporated into an application by being deployed to a J2EE or .NET application server, or embedded directly in an application.

\section{Business Rules Application for CRM}

The most promising domain for application of business rules software at the current time is Customer Relationship Management (CRM). CRM is a business strategy, directed at the sustainable business building, the kernel of which is "client-oriented" approach. CRM also includes technology of customer retention and client-enterprise interaction history database maintenance. This strategy based on collecting information about clients at all stages of service life cycle, extracting knowledge from it and using this knowledge for business amelioration.

In this paper we will consider how business rule management conception can be applied to one of the Russian enterprise - Podolsk (town) Electromechanical Plant PEMP (http://www.i-mash.ru/predpr/1250), which is producing hydraulic equipments. This enterprise is specialized in the supply of hydraulic and pneumatic equipment for energy companies, construction, transportation and industrial complex. The firm is producing various hydraulic equipment (pumps, motors, etc.), designed for application in marine hydraulics, transportation systems, facilities for the repair of wells, as well as railway equipment (locomotives). All the necessary spare parts and rubber products for this equipment are also manufactured .

The problem, nowadays facing the company, consists from reducing the amount of orders for products and customers churn. In modern conditions the market is saturated with offerings, and the struggle for the client retention becomes one of the major problems of each company. Analysis of customers data, selecting different customer categories and corresponding business rules, providing to customers by services, relevant to the customer's consumption profile. Flexible system of discounts and offers for client will allow the company to increase sales, identify and select the most valuable and reliable customers and, accordingly to their score, focus on them, as result to improve the sales department efficiency and business in general.

Business rules manually could be derived from several sources, and the mainly three sources are described as following policy statements and objectives of organization, business process and external factors (e.g. laws and regulations). The tracing the sources of business rules can help to the personnel to discover the need of changing BR. If the content of the source is changing, the rule relating to this source has to be modified or removed. 
Business rules can be described in simple natural language or by mean decision tables or decision trees. If simple natural language would be chosen the rules must be readable and accessible to all interested parties: business owner, business analyst, technical architect, and so on

According to business rules for each category of customers the company is offering different set of products and services according to different customer's profile.

Table 1. PEMP customer relationship business rules

\begin{tabular}{|c|c|c|}
\hline Business rule & Formalized rules & Source Type \\
\hline $\begin{array}{l}\text { If the volume of sales order from } \\
80,000 \text { to } 120,000 \text { rubles and the } \\
\text { status of the client "risk", then } \\
\text { give a discount of } 5 \%\end{array}$ & $\begin{array}{c}\text { IF } 80000<\text { order_value }<120000 \text { and } \\
\text { status = "bad" THEN set discount } 5 \% \text { IF } \\
80000<\text { order_value }<120000 \text { and status = } \\
\text { "bad" THEN set discount of } 5 \%\end{array}$ & $\begin{array}{c}\text { Corporate } \\
\text { policies, dynamic }\end{array}$ \\
\hline $\begin{array}{l}\text { If the volume of customer order } \\
\text { less than } 80000 \text { rubles, then do } \\
\text { not give a discount }\end{array}$ & $\begin{array}{c}\text { IF order_value }<80000 \text { THEN set discount } \\
0 \% \text { IF order_value }<80000 \text { THEN set } \\
\text { discount } 0 \%\end{array}$ & $\begin{array}{l}\text { Corporate } \\
\text { policies, dynamic }\end{array}$ \\
\hline $\begin{array}{l}\text { If the industry of the client } \\
\text { equipment, then to appoint a } \\
\text { corporate rating of } 2\end{array}$ & $\begin{array}{c}\text { IF otras } 1=" 1 " \text { THEN score }:=\text { score }+2 \text { IF } \\
\text { otras } 1=" 1 " \text { THEN score }:=\text { score }+2\end{array}$ & $\begin{array}{c}\text { Corporate policy, } \\
\text { static }\end{array}$ \\
\hline $\begin{array}{l}\text { If the industry of the client } \\
\text { shipbuilding, then assign a } \\
\text { corporate rating of } 8\end{array}$ & $\begin{array}{c}\text { IF otrasl = "3" THEN score }:=\text { score }+8 \text { IF } \\
\text { otrasl }=" 3 " \text { THEN score }:=\text { score }+8\end{array}$ & $\begin{array}{c}\text { Corporate policy, } \\
\text { static }\end{array}$ \\
\hline $\begin{array}{l}\text { If the term of the client in this } \\
\text { sector from } 11 \text { to } 20 \text { years, to } \\
\text { appoint a corporate score equal to } \\
14\end{array}$ & $\begin{array}{c}\text { IF } 11<\text { srok<20 THEN score:=score+14 IF } \\
11<\text { srok <20 THEN score: }=\text { score }+14\end{array}$ & $\begin{array}{l}\text { Corporate policy, } \\
\text { static }\end{array}$ \\
\hline $\begin{array}{l}\text { If a corporate customer rating } \\
\text { greater than } 25 \text {, then a status of } \\
\text { reliable customer }\end{array}$ & $\begin{array}{c}\text { IF score }>25 \text { THEN status:="good" IF } \\
\text { score }>25 \text { THEN status: = "good" }\end{array}$ & $\begin{array}{c}\text { Corporate policy, } \\
\text { static }\end{array}$ \\
\hline
\end{tabular}

We have been elaborated the business rules of this enterprise in the next stages:

Defining the main sources of business rule analyses,

$>$ Rules formalizing and catalog creating,

$>$ BRMS market analyses and choosing appropriate system,

$>$ Creating a model in the BRMS environment,

$>$ Elaborating database and store products and customers data,

$>$ Testing the model,

$>$ Integration with EIS.

As a result of this work hundreds of business rules were selected, several examples of which are presented in the Table 1. 
We suppose that the business model, based on business rules, makes possible for the plant quickly adjust production and services to changing conditions of market environment by mean procedure of clients clustering according to their significance. The results of such clustering are used for risk rating evaluation and for using flexible discount system, that makes possible to held profitable clients from churn to another companies and eventually for providing to the company additional profit.

In our work for experimental research BRMS Visual rules Modeler was selected and installed. The next structure of the database, containing products and customer data, was developed (figure 1).

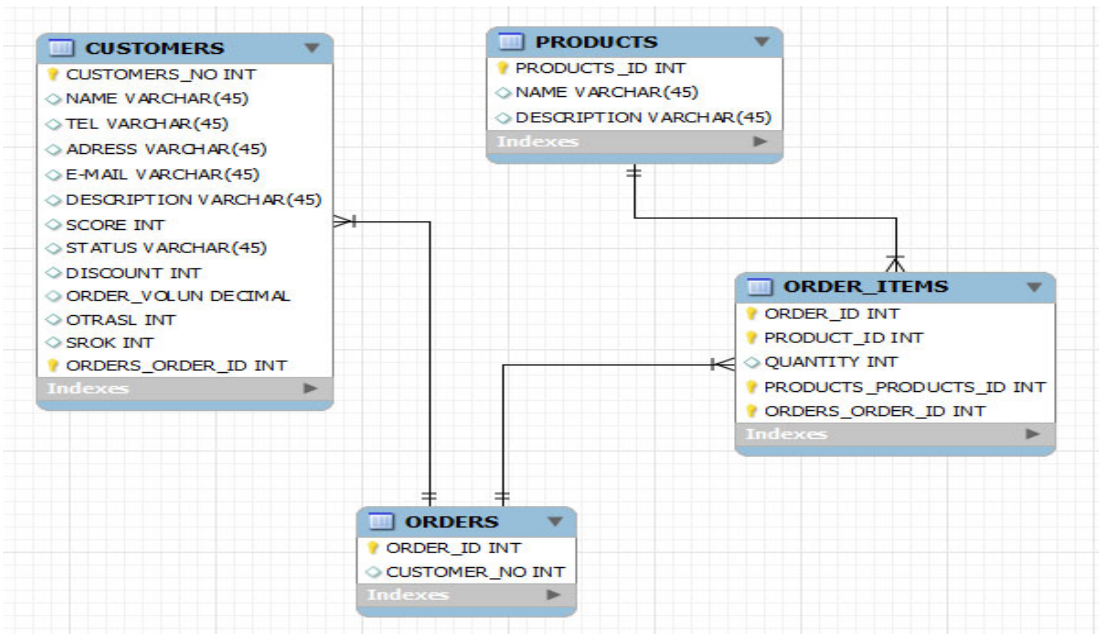

Fig. 1. Database structure

In this data base table «CUSTOMERS» contains the clients legal entities data such as title, description, legal address, phone number, email address, branch of the enterprise, the term of operation of the client on the market, besides the table contains several computed field to store such data as score, the volume of orders and size of the discounts computed as a result of the business rules application. Data about customer's orders are saved in tables «ORDER» and «ORDER_ITEMS». Table «PRODUCTS» contains the hydraulic products items catalog company.

With application Visual Rules Modeler was created business rules stream "Discount Assign", depending from order volume and client status. Stream rules "Discount Assign" illustrates the rule set, which is assigning discounts to customers. Business Rules allow management to create a flexible system of discounts, value which is easy to modify (figure 2).

As can be seen in the data base and views into data fields score, status, and the order volume discounts values have been inserted in accordance with the developed model business rules for certain customer and logic of the discounts assignment decision to the different users. So through the developed business rules model, companies can quickly adapt to changing environmental conditions and dynamic competitive environment. 


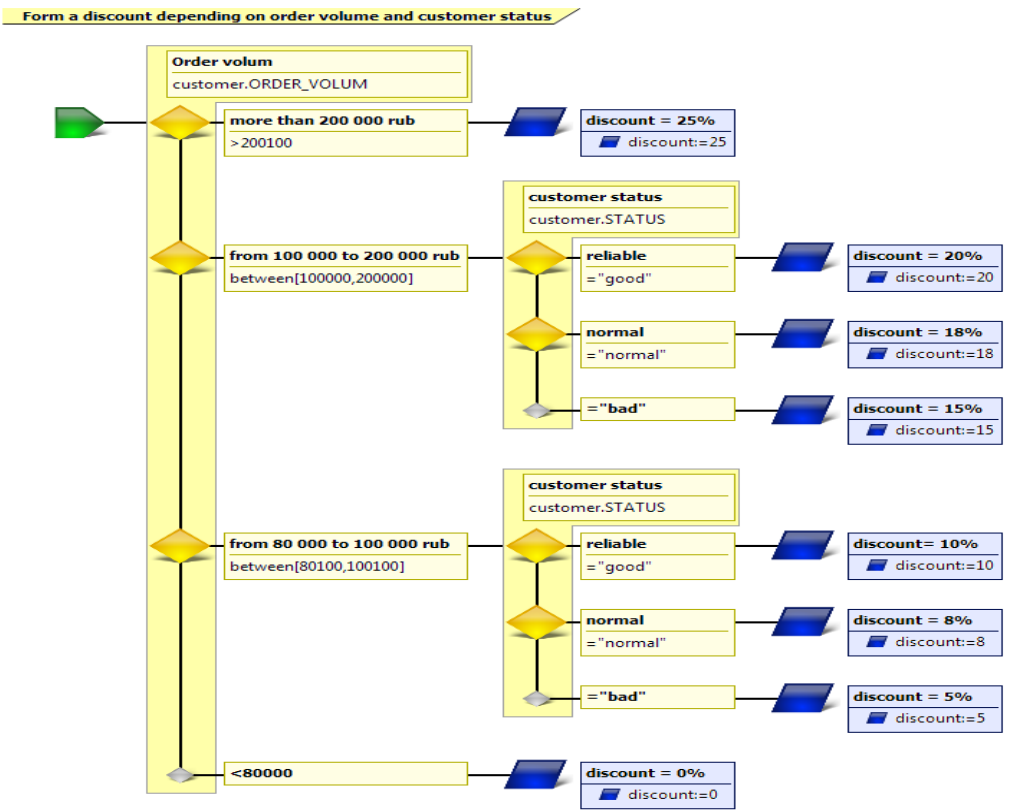

Fig. 2. Business Rules, that assigned the discounts to the clients

The data, prepared for the model testing, which were loaded to the data base and then the discount percents values were computed according business rules to different customers are presented at figure 3.

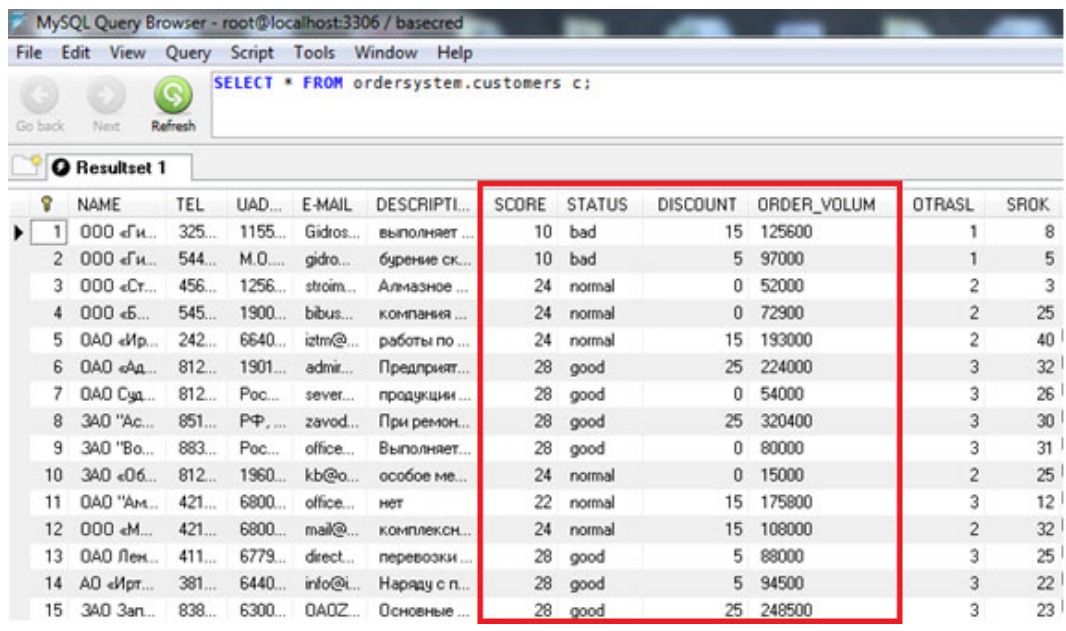

Fig. 3. Clients discount percents data, computed according business rules 
Rules stream at figure 4 shows that business rules stream is actually displayed as graphic interface for making the program, containing as business rules for conditions checking as data base SQL commands, performing in the definite order.

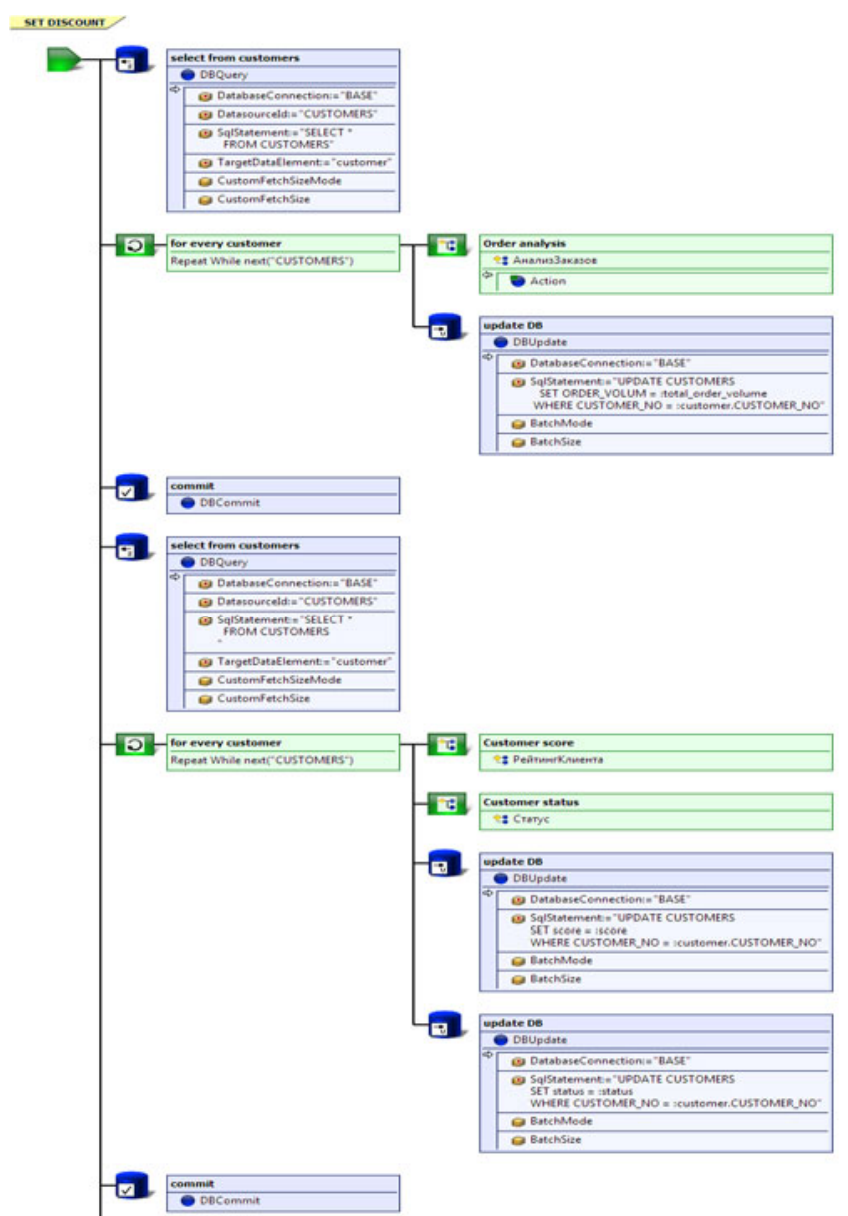

Fig. 4. Rule stream as a program, containing business rules and SQL operators

\section{Rules Discovery by FCA Method}

One of the problems which confronts business analytics is problem of business rules discovery. This problem is very hard and has very high dimension. The data mining method that may be used for extracting rules from client and services data (figure 5). 


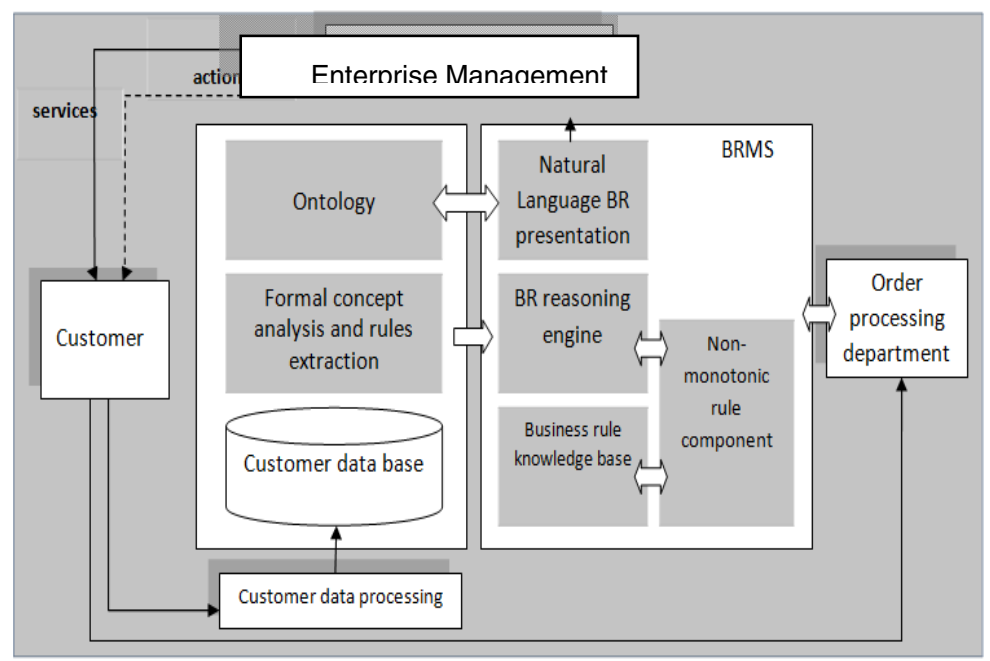

Fig. 5. Adaptive EIS, self-adjusting to the customers' consumption profile and motivation customers to increase volume of consumption

At the figure 5 is presented adaptive EIS, where feedback loop used for adjusting EIS services on the base of analyzing customer's data and applying the results for correcting services and products to customer consumption profile. The formal concept analysis is one of such method. Formal concept analysis was primary proposed by R. Wille in 1982 year [8] and it finds its application at the different domains for discovering data structure.

Aside from selection clients group and its visualizing this method provide possibility for search attributes dependencies in form of implications. The clients may be regarded as objects and their personal data, kinds of services, intensity services consumption and performed with them marketing actions as a attributes, which customers have. Upon this data, the set of objects with common attributes values may be discovered.

The data are presented as formal context that is as a table, the rows of which correspond to object and the columns - to attributes. If some object has definite attribute value then at the intersection is placed one (or cross). The essence of this method consists in the next [9]. Formal context $K:=(G, M, I)$ consists from sets $G, M$ and a binary relation $I \subseteq G \times M$, where $M$-attribute set, $G$-objects sets, expression $(g, m) \in I$ - signifies that object $\mathrm{g}$ has attribute $m$.

Formal context may be presented as binary matrix, rows of which correspond to object and column - to attributes values. Let us define the mappings for $A \subseteq G$, $B \subseteq M$ :

$\phi: 2^{M} \rightarrow 2^{G} \quad u \psi: \quad 2^{G} \rightarrow 2^{M}$ $\phi(A)=\operatorname{def}\{m \in M \mid g \operatorname{Im} \forall g \in A\}, \psi(B)=\operatorname{def}\{g \in G \mid g \operatorname{Im} \forall m \in B\}, A \subseteq G$, $B \subseteq M$. 
$\operatorname{pair}(A, B)$ - is named as a formal concept of context $K$, if $\phi(A)=B, \psi(B)=A$ (or another notation: $A^{\prime}=B$, and $B^{\prime}=A$ ). The object set $A$ is named as extent and attribute set $B$ - intent of the concept. Therefore, the formal concept it is a set of objects and corresponding attributes, such that every object has all attributes from the attribute set. In case, when extent of concept $C_{2}$ is included into the extent of concept $C_{1}$, that is $\operatorname{Ext}\left(C_{2}\right) \subseteq \operatorname{Ext}\left(C_{1}\right)$, we say that $C_{1}$ is a superconcept and $C_{2}-$ is subconcept. The concepts hierarchy is defined by relation subconcept-superconcept:

$$
\left(A_{1}, B_{1}\right) \leq\left(A_{2}, B_{2}\right) \Leftrightarrow A_{1} \subseteq A_{2}\left(\Leftrightarrow B_{1} \supseteq B_{2}\right) .
$$

For formal context $(G, M, I)$ and $\mathrm{X} \subseteq G, S \subseteq M$,

1. $X_{1} \subseteq X_{2} \Rightarrow X_{1}^{\prime} \supseteq X_{2}{ }^{\prime}$ для $X_{1}, X_{2} \subseteq G$,

2. $S_{1} \subseteq S_{2} \Rightarrow S_{1}{ }^{\prime} \supseteq S_{2}{ }^{\prime}$ для $S_{1}, S_{2} \subseteq M$,

3. $X \subseteq X^{\prime \prime}$ и $X^{\prime}=X^{\prime \prime \prime}$ для $X \subseteq G$,

4. $S \subseteq S^{\prime \prime}$ и $S^{\prime}=S^{\prime \prime \prime}$ для $S \subseteq M$.

5. $X \subseteq S^{\prime} \Leftrightarrow S \subseteq X^{\prime}$.

The ordered set of all formal concepts of $(G, M, I)$ is denoted by $L(G, M, I)$ and is called the concept lattice of $(G, M, I)$. Infimum and supremum of the $L(G, M, I)$ are given by:

$$
\begin{aligned}
& \bigwedge_{j \in J}\left(X_{j}, S_{j}\right)=\left(\bigcap_{j \in J} X_{j},\left(\bigcup_{j \in J} S_{j}\right)^{\prime \prime}\right), \\
& \vee_{j \in J}\left(X_{j}, S_{j}\right)=\left(\left(\bigcup_{j \in J} X_{j}\right)^{\prime \prime}, \bigcap_{j \in J} S_{j}\right) .
\end{aligned}
$$

The most important problem is: how to build the concept lattice for the context $(G$, $M, I)$. We obtain the most simple answer, creating $\left(X^{\prime \prime}, X^{\prime}\right)$ for all $X \subseteq G$ or $\left(S^{\prime}, S^{\prime \prime}\right)$ for all $S \subseteq M$.

In practice, in this paper we use the program Concept Explorer (http:// sourceforge.net/projects/conexp/). The context for our PEMP example is depicted at

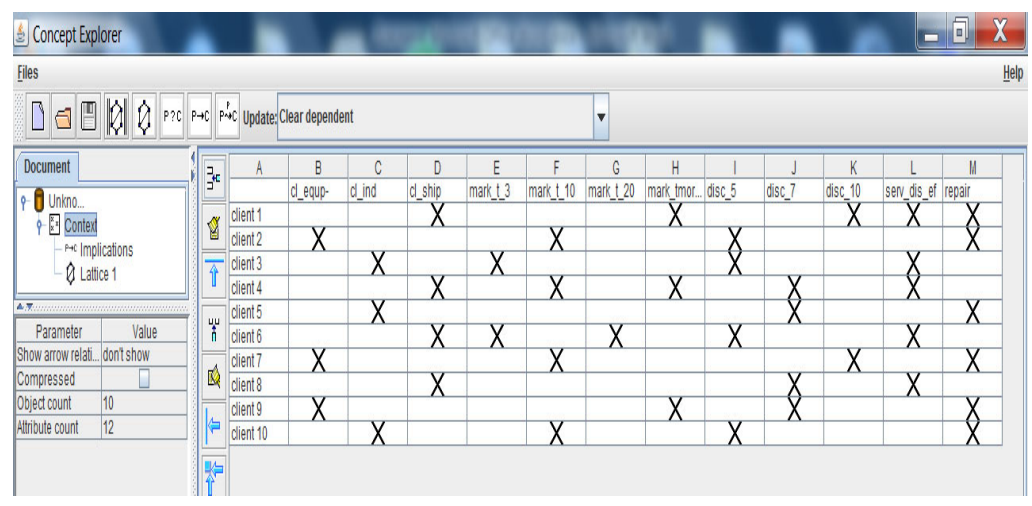

Fig. 6. The context, describing customers of the PEMP 
figure 6, where data about customers, their consumption profile, values of score for clients, the efficiency data of preferences and discounts are contained.

The concept lattice for our case is presented at figure 7. This lattice provides us an opportunity to explore and interpret the relationship between concepts.

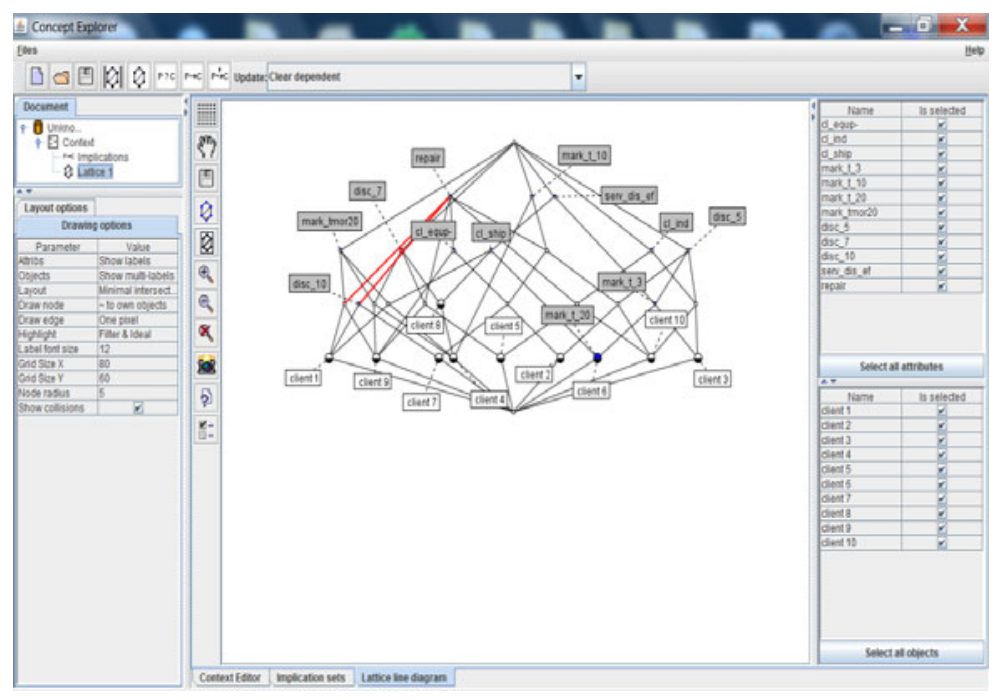

Fig. 7. The concept lattice

The dependency notion between attributes is based on the next idea: if for all objects in the context, for which some property $P$ is true, some another property $C$ is also true, then implication $P \rightarrow C$ is valid. More precisely, the implication $P \rightarrow C$ is valid in a context $K=(G, M, I)$, where $P \subseteq M$ and $C \subseteq M$ iff $C \subseteq P^{\prime \prime}$.

\section{$5 \quad$ Rules Quality Criteria}

The implication means that all objects of context which contain attributes $P$ also contain attribute $C$. That is in the situation $P$ manager must make decision $C$. Let us define the next measures of rules quality:

- Support: $\operatorname{supp}(P)=\operatorname{Card}(\psi(P) / \operatorname{Card}(G)-$ the rate of objects, containing nonempty values of attributes $\mathrm{P}$, comparing to all number of objects.

- Confidence: $\operatorname{conf}(\mathrm{P} \Rightarrow C)=\operatorname{supp}(P \cup C) / \operatorname{supp}(P)$,

- Lift: $\operatorname{lift}(P \Rightarrow C)=\operatorname{supp}(P \cup C) / \operatorname{supp}(P) \times \operatorname{supp}(C)$,

- Conviction : $\operatorname{conv}(P \Rightarrow C)=1-\operatorname{supp}(C) / 1-\operatorname{conf}(P \Rightarrow C)$.

The implication set, discovered by Concept explorer, are business rules, some example of which are presented below. Business rules with confidence 100\%: 
- IF branch of the client = equipment THEN repair_discount_5\%;

- IF time on a market $>10$ THEN repair_discount_5\%;

- IF time on a market > 20 THEN repair_discount_10\% and service_discount and consulting_free;

- IF branch of client = shipment AND time on market> 20 THEN serv_discount AND sale_discount $=10 \%$ AND repair_discount.

The rules with confidence less than $100 \%$ :

- IF branch of client = shipment THEN serv_discount; $($ confidence $80 \%)$

- IF branch of client = industry THEN consulting free; $($ confidence $60 \%)$

We assume that data may be incomplete or contradictory and therefore implication derived by Conexp software should be considered within the frame of non-monotonic logic, and realized as defeasible theory rules. Defeasible logic is practical nonmonotonic logic, containing facts, strict rules, defeasible rules and supporting relations.

Non-monotonic reasoning is an approach that allows reasoning process with incomplete or changing information. More specifically, it provides mechanisms for taking back conclusions that, in the presence of new information, turn out to be wrong and for deriving new, alternative conclusions instead.

The non-monotonic subset of the rules is obtained by computing the lattice corresponding to the subcontext consisting of the original context without those attributes which do not apply to the set of all objects. FCA computes the minimal base of implications corresponding to the actual context and asks the user if each single implication is valid in the universe of objects or if a counterexample is known. The counterexamples are than added to the context and the implications are newly computed until all implications are accepted.

\section{Conclusions}

In this paper we have considered the conception of adaptive EIS, which has possibility of tuning its business rules set by mean customer's data mining with applying formal concept method. This approach is especially important when number of business rules, which are changed with time, counts by thousands.

We have shown that on the way of including into the structure of modern EIS means for automated decision making, the essential role may play Formal Concept Analysis method, which can help to find out specific dependencies between observed customers' data and services, provided by business, usability data.

The next step may be research the adaptive EIS with varying structure, including data base structure, procedures and semantic concepts of language, describing the domain of application. 


\section{References}

1. Romanov, V., Veynberg, R., Polujectova, A.: Customer-Telecommunications Company's Relationship Simulation Model (RSM), Based on Non-Monotonic Business Rules Approach and Formal Concept Analysis Method. In: SpringSim 2011, p. 54. Program Book, Boston (2011)

2. What IS Enterprise Decision Management or EDM? FICO Decision Management Blog, http: / /dmblog.fico.com/2006/06/what_is_enterpr_1.html

3. Will Automated Decision Support Tools Replace the Business Analytics? http: / / thinkup. waldenu. edu/finance-and-accounting/financialbusiness-systems/item/11860-will-automated-decision-supporttools-replace-business-analytics

4. Oracle Business Rules Overview, http: / /www. oracle.com/technetwork/.../business-rules/.../ index-085313.html

5. SAP Netweaver Business Rules overview \#sapteched09 - JT on EDM, http: / / jtonedm.com/2009/10/13/sap-netweaver-business-rulesoverview-sapteched09/

6. Microsoft BizTalk Server Business Rule Framework, http: / /www. microsoft.com/biztalk/.../business-ruleframework. aspx

7. IBM ILOG JRules | ILOG JRules Tutorial | ILOG JRules Interview ..., http: / /www. javagenious.com/... /ILOG-JRULES-TUTORIALS

8. Wille, R.: Restructuring lattice theory: an approach based on hierarchies of concepts. In: Rival, I. (ed.) Ordered Sets, pp. 445-470. Reidel, Dordrecht (1982)

9. Ganter, B., Stumme, G., Wille, R. (eds.): Formal Concept Analysis Foundation and Applications, p. 349. Springer, Heidelberg (2005) 